\title{
Mecanismos da Dor Nociceptiva em Indivíduos com Artrite Reumatóide: uma revisão sistemática
}

\author{
Acadêmica do $4^{\circ}$ ano do curso de Fisioterapia da \\ Escola Bahiana de Medicina e Saúde Pública \\ Maria Gabriela Silva Hage Carmo \\ (Endereco Institucional: Av. Dom João VI 275, \\ Brotas, 40 290-000 Salvador-BA, Brazil, \\ cpsaraujo1@hahiana.edu.br) \\ Gabriela Carneiro Costa \\ Acadêmica do 49 ano do curso de Fisioterapia da \\ Escola Bahiana de Medicina e Saúde Pública \\ Fisioterapeuta, mestre em Ciências Morfológicas \\ Cintia Silveira Araújo Pinheiro \\ pela UFRJ, professora da Escola Bahiana de Medicina \\ e Saúde Pública, Salvador, Bahia, Brasil \\ Tipo de Pesquisa Revisão da Literatura \\ Origem da Pesquisa Trabalho de Conclusão de Curso de Fisioterapia da \\ Escola Bahiana de Medicina e Saúde Pública \\ Análise do Manuscrito Corpo Editorial da Bahiana \\ Tipo de Análise do Manuscrito Peer Blind Review \\ Recebido em Mai/11 Aprovado em Jun/11
}

\section{Resumo}

Introdução: A Artrite Reumatóide (AR) é uma doença inflamatória crônica, de etiologia desconhecida, caracterizada por inflamação articular, dor e déficit funcional. Como implicações da doença, os indivíduos podem apresentar fadiga, diminuição da amplitude de movimento e dor. A dor parece ser o sintoma mais importante para a maioria dos pacientes, sendo considerado o principal objetivo da maior parte dos tratamentos reumatológicos. Objetivo: identificar na literatura os mecanismos da dor nociceptiva em indivíduos com Artrite Reumatóide. Métodos: Foram acessadas as bases de dados: Medline, Cochrane e SciELO no período de 10 a 30 de abril de 2011. De acordo com as palavras-chave encontradas nos títulos e resumos dos estudos, foram incluídos aqueles que fizeram referência às causas e mecanismos da dor nociceptiva na AR e estavam publicados no idioma português, inglês e espanhol. Foram excluídos os estudos que se referiram apenas ao tratamento da AR e os que não descreveram a metodologia e/ou não apresentaram a sessão de resultados e discussão. Resultados: Foram selecionados 32 artigos para a leitura na íntegra, sendo 22 excluídos pela metodologia ou conteúdo incompatível com o tema proposto. Foi evidenciado que fatores como TNF- $\alpha$, quimiocinas, citocinas, prostanóides, COX-2, PKC, neutrófilos e canal TRPV1 são as principais substâncias relacionadas à manifestação clínica álgica nociceptiva da AR. Conclusão: Neste estudo pôde-se observar que diversas substâncias desempenham um papel relevante para a potencialização da dor nociceptiva na AR através de diferentes mecanismos. Dessa forma evidenciou-se a importância de um tratamento multidisciplinar, abordando os diversos aspectos da dor, minimizando ou retardando as amplificações da dor no SNC.

Palavras-chave: Artrite Reumatóide, desordens reumatológicas; dor nociceptiva 


\title{
Mechanisms of nociceptive pain in people with rheumatoid arthritis: a systematic review
}

\begin{abstract}
Introduction: Rheumatoid arthritis (RA) is a chronic inflammatory disease of unknown etiology, characterized by joint inflammation, pain and functional deficit. As implications of the disease, individuals may experience fatigue, decreased range of motion and pain. The pain seems to be the most important symptom for most patients and is considered the main goal of most rheumatic treatments. Objective: To identify the mechanisms in the literature of nociceptive pain in patients with rheumatoid arthritis. Methods: We accessed the databases: Medline, SciELO and Cochrane in the period from 10 to 30 April 2011. According to the keywords found in titles and abstracts of the studies included were those who made reference to the causes and mechanisms of nociceptive pain in RA and were published in Portuguese, English and Spanish. We excluded studies that reported only to the treatment of RA and those who did not describe the methodology and / or did not present the results and discussion session. Results: We selected 32 articles to read in full, 22 being excluded by the methodology or content inconsistent with the theme. It was shown that factors such as TNF$\alpha$, chemokines, cytokines, prostanoids, COX-2, PKC, neutrophils and TRPV1 channels are the main substances related to nociceptive algic clinical manifestation of RA. Conclusion: In this study it was observed that several substances play an important role for the potentiation of nociceptive pain in RA through different mechanisms. Thus it became clear the importance of a multidisciplinary approach, addressing the various aspects of pain, minimizing or delaying the amplification of pain in the CNS.
\end{abstract}

Keywords: Rheumatoid arthritis, rheumatic disorders, nociceptive pain

\section{Los mecanismos del dolor nociceptivo en personas con artritis reumatoide: una revisión sistemática}

\section{Resumen}

Introducción: La artritis reumatoide (AR) es una enfermedad inflamatoria crónica de etiología desconocida, caracterizada por la inflamación de las articulaciones, dolor y déficit funcional. Como las consecuencias de la enfermedad, las personas pueden sentir fatiga, disminución de la amplitud de movimiento y dolor. El dolor parece ser el síntoma más importante para la mayoría de los pacientes y se considera el objetivo principal de la mayoría de los tratamientos reumáticos. Objetivo: Identificar los mecanismos de la literatura del dolor nociceptivo en pacientes con artritis reumatoide. Métodos: Se accede a la base de datos: Medline, SciELO y Cochrane en el período 10-30 de abril 2011. De acuerdo con las palabras clave en los títulos y resúmenes de los estudios incluidos fueron los que hizo referencia a las causas y mecanismos del dolor nociceptivo en la AR y fueron publicados en Portugués, Inglés y Español. Se excluyeron los estudios que informaron sólo para el tratamiento de la RA y los que no describe la metodología y / o no presentar los resultados y sesión de discusión. Resultados: Se seleccionaron 32 artículos para leer en su totalidad, 22 se excluyeron por la metodología o contenidos incompatibles con el tema. Se ha demostrado que factores como el TNF- $\alpha$, quimiocinas, citocinas, prostaglandinas, la COX-2, PKC, los neutrófilos y los canales TRPV1 son las principales sustancias relacionadas con la manifestación nociceptivo álgicas clínica de la AR. Conclusión: En este estudio se observó que varias sustancias juegan un papel importante para la potenciación del dolor nociceptivo en la AR a través de mecanismos diferentes. Así se puso de manifiesto la importancia de un enfoque multidisciplinario, abordar los diferentes aspectos del dolor, minimizar o retrasar la amplificación del dolor en el SNC.

Palabras-clave: Artritis reumatoide, enfermedades reumáticas, dolor nociceptivo 
Mécanismes de la douleur nociceptive chez les personnes atteintes de polyarthrite rhumatoïde: une revue systématique

\section{Résumé}

Introduction: La polyarthrite rhumatoïde (PR) est une maladie inflammatoire chronique d'étiologie inconnue, caractérisée par l'inflammation des articulations, des douleurs et déficit fonctionnel. Comme conséquences de la maladie, les personnes peuvent ressentir de la fatigue, diminution de l'amplitude du mouvement et la douleur. La douleur semble être le symptôme le plus important pour la plupart des patients et est considéré comme l'objectif principal de la plupart des traitements rhumatismales. Objectif: identifier les mécanismes de la littérature de la douleur nociceptive chez les patients atteints de polyarthrite rhumatoïde. Méthodes: Nous avons accédé à la base de données: Medline, Cochrane SciELO et dans la période de 10 à 30 avril 2011. Selon les mots-clés trouvés dans les titres et les résumés des études incluses ont été ceux qui ont fait référence à des causes et des mécanismes de la douleur nociceptive dans la PR et ont été publiés en portugais, anglais et espagnol. Nous avons exclu les études qui ont déclaré que pour le traitement de la PR et ceux qui n'ont pas de décrire la méthodologie et / ou ne présente pas les résultats et la séance de discussion. Résultats: Nous avons sélectionné 32 articles à lire en entier, 22 étant exclus par la méthodologie ou du contenu incompatible avec le thème. Il a été montré que des facteurs tels que le TNF- $\alpha$, les chimiokines, les cytokines, prostanoïdes, inhibiteurs de la COX-2, la PKC, les neutrophiles et les canaux TRPV1 sont les principales substances nociceptives liées à la manifestation clinique de la PR algiques. Conclusion: Dans cette étude, on a observé que plusieurs substances jouent un rôle important pour la potentialisation de la douleur nociceptive dans la PR par des mécanismes différents. Ainsi il est devenu clair sur l'importance d'une approche multidisciplinaire, abordant les différents aspects de la douleur, en minimisant ou en retardant l'amplification de la douleur dans le SNC.

Mots-clés: Polyarthrite rhumatoïde, les maladies rhumatismales, douleurs nociceptives

\section{Introdução}

A Artrite Reumatóide (AR) é uma doença inflamatória crônica, caracterizada por inflamação articular, dor1 e déficit funcional.2,3 Possui etiologia ainda desconhecida, porém inadequadas respostas auto-imunes ao estresse parecem desempenhar um relevante papel nessa inflamação crônica.4 Acomete duas a três vezes mais o sexo feminino e há um aumento da prevalência com a idade.5,6,7 Estudos epidemiológicos estimam a prevalência de AR em aproximadamente 0,5 a 1\% na população mundial e 0,2 a 1\% no Brasil.7,8 0 seu diagnóstico depende da associação de sinais e sintomas clínicos e achados laboratoriais e radiográficos, baseados no critério de classificação do American College of Rheumatology (ACR). 5

Sabe-se que como implicações da doença, indivíduos portadores de AR podem apresentar fadiga, diminuição da amplitude de movimento, diminuição da flexibilidade muscular e dor9. A dor parece ser o sintoma mais importante para a maioria dos pacientes, levando a crer que a melhora desse quadro é considerada o principal objetivo da maior parte dos tratamentos reumatológicos. 10

Por isso a relevância de estudar esta sintomatologia tão presente nesses indivíduos e principalmente pela possibilidade de serem elaboradas novas bases teóricas que possam fundamentar e direcionar novos tratamentos. 0 presente estudo tem como objetivo identificar na literatura os mecanismos da dor nociceptiva em indivíduos com Artrite Reumatóide (AR). 


\section{Material \& Método}

\section{Tipos dos estudos}

Essa revisão sistemática incluiu todos os estudos experimentais, descritivos ou ensaiosclínicos encontrados. Aqueles que não apresentaram descrição da metodologia foram excluídos.

Métodos para identificação dos estudos (estratégia de busca)

A triagem em base de dados eletrônicas de livre acesso foi feita no período de 10 a 30 de abril de 2011, utilizando os termos em dois idiomas: português "artrite reumatóide", "desordens reumatológicas"; "dor nociceptiva" e inglês "rheumatoid arthritis"; "rheumatic diseases"; "rheumatic disorders"; "nociceptive pain".

Bases de dados utilizadas: Medline; Cochrane; Scielo.

\section{Coleta e análise}

Na triagem buscou-se os títulos e resumos dos estudos de acordo com as palavras-chave pré-estabelecidas.

Critérios de inclusão

- Estudos que fizeram referência às causas e mecanismos da dor nociceptiva na AR;

- Artigos publicados até abril de 2011.

Critérios de exclusão

- Estudos que se referiram apenas ao tratamento da AR;

- Estudos que não descreveram a metodologia e/ou não apresentaram a sessão de resultados e discussão, por serem considerados trabalhos de baixo poder científico.

\section{Critérios para análise metodológica dos estudos}

Para avaliação da qualidade metodológica utilizou-se os critérios da escala de avaliação proposta por JADAD11. Foi dado um escore de uma a oito cruzes de acordo com a presença dos seguintes itens relacionados:

- Randomização, Cegamento, Validado, Objetivo e hipóteses claras, Critérios de Inclusão e Exclusão claros, Descrição da investigação, Descrição da intervenção, Análise adequada, Descrição do método e Objetivo alcançado

Em cada artigo foram coletadas informações sobre os mecanismos da dor nociceptiva e os resultados sintetizados em forma de tabela.

\section{Resultados}

Ao serem utilizadas todas as palavras-chave, identificou-se 103 títulos e resumos, incluindo 11 repetições. Foram selecionados 32 estudos para serem lidos na íntegra, sendo que 5 foram excluídos por não descreverem a metodologia e 13 foram excluídos porque o conteúdo não se aplicou ao objetivo dessa pesquisa. 0 resultado foi apresentado em forma de fluxograma (Figura 1), conforme proposto por Pai et al. (2007).12

Foram incluídos no estudo, portanto, 10 artigos, sendo 6 experimentais e 4 ensaiosclínicos. De acordo com as informações coletadas nos artigos selecionados, uma análise da qualidade metodológica foi realizada (Quadro I). Observou-se que apenas dois trabalhos alcançaram todos os critérios adotados para análise da qualidade metodológica dos artigos, podendo-se verificar a pouca utilização dos critérios como randomização, cegamento e comparação entre placebo/sham. 
Os resultados encontrados nesta revisão foram sistematizados por substâncias, para uma melhor compreensão. A síntese das informações sobre os mecanismos da dor nociceptiva encontrados foram dispostas na Tabela 1.

- TNF- $\alpha$ como importante estratégia de tratamento e sua relação como canal TRPV1, PKC, COX-2, IL-1ße neutrófilos

Russel et al. (2009) fizeram um estudo cujo o objetivo foi avaliar o efeito inflamatório do fator de necrose tumoral-alfa (TNF- $\alpha$ ) induzida na hiperalgesia bilateral. Além da comprovação desta hipótese, foi visto que esta hiperalgesia provocada pelo TNF- $\alpha$ é dependente dos canais de receptor potencial transitório vanilóide1 (TRPV1), devido ao seu significante aumento neste processo inflamatório. Associado a isso, sabe-se que a proteína quinase-C (PKC) potencializa o TRPV1, diminuindo o nível de calor local, pois este canal se fecha a altas temperaturas $\left(>45^{\circ} \mathrm{C}\right)$. 0 estudo encontrou que a injeção de pequenas doses de TNF- $\alpha$ não obteve significante efeito na hiperalgesia, porém, altas doses a provocou bilateralmente. Para comprovar o papel da PKC na inflamação, utilizou-se um inibidor de PKC juntamente à administração de TNF- $\alpha$ e foi observado bloqueio da hiperalgesia bilateralmente. Ipsilateralmente, a ciclo-oxigenase 2 (COX-2) derivada da prostaglandina E (PGE) também desempenha um papel essencial na hiperalgesia. Para o desenvolvimento e manutenção desta inflamação, foi comprovada a presença da interleucina 1 beta (IL-1ß) e neutrófilos nestes respectivos processos. 12

Hess et al. (2011) estudaram o efeito do bloqueio do TNF- $\alpha$ como estratégia para o tratamento da AR. Foi observado através da Ressonância Magnética funcional (RMf) no Sistema Nervoso Central (SNC) a diminuição do fluxo sanguíneo para áreas envolvidas na percepção da dor, como tálamo, córtex somatosensorial, sistema límbico, córtex cingulado e ínsula nas primeiras $24 \mathrm{~h}$ de administração do bloqueador. Esses achados indicaram que o TNF- $\alpha$ pode estar relacionado à função de estruturas cerebrais envolvidas tanto com a emoção como na percepção da dor.13

- Citocinas, quimiocinas, neutrófilos, aminas simpatomiméticas possuem um importante papel na hipernocicepção inflamatória

Pinto et al. (2010) observaram o papel da interleucina 17 (IL-17) na nocicepção articular em um modelo de AR mBSA induzido. Para avaliar a participação da IL-17 na gênese da dor, foi utilizado um antagonista da IL-17 e observou-se diminuição da hipernocicepção articular e do recrutamento de neutrófilos que são associados a este modelo de AR induzido. Foi visto também que a injeção intra-articular de IL-17 induz hipernocicepção e recrutamento neutrofílico, além da expressão de TNF- $\alpha$, IL-1ß e da quimiocina CXCR1/2. Por outro lado, 0 bloqueio desses três mediadores bloqueou os efeitos da IL-17. A injeção de IL-17 induziu ainda ao aumento da expressão de COX-2 e de prostaglandinas no exsudato, aumentando a inflamação e diminuindo o limiar nociceptivo. ${ }^{14}$

Constandil et al. (2009) fizeram um estudo experimental que visou investigar a contribuição das células da glia e os efeitos do IL-1ß na transmissão nociceptiva pela medula espinal, em um grupo com artrite induzida e outro controle. Para a interrupção da função glial foi administrado propentofilina. Observou-se que o limiar de ativação das fibras-C foi menor nos ratos com monoartrite induzida e com tratamento placebo. Já os ratos normais com indução de propentofilina obtiveram o maior limiar. A aplicação de 12 estímulos consecutivos de $1 \mathrm{~Hz}$ induziu o Wind-up em todos os grupos, porém, os grupos que receberam tratamento obtiveram um maior Wind-up após a aplicação de IL-1ß. Portanto, parece existir a participação da IL-1ß na hipernocicepção e o tratamento com propentofilina ao inibir a função da célula da glia, inibe também a ação do IL-1ß.15

- Expressão de neutrófilos durante a inflamação tecidual periférica

Mitchell et al. (2008) estudaram a expressão gênica em um estado inflamatório periférico parecido ao que ocorre na AR. Após a indução de inflamação articular com carragenina, foi demonstrado aumento da expressão dos genes S100A8 e S100A9 em neutrófilos e monócitos presentes nos vasos do cérebro, da glândula pituitária e do segmento 
da medula espinal correspondentes ao membro inflamado. Esses achados sugerem a interação entre uma subpopulação de leucócitos e o Sistema Nervoso Central (SNC) na inflamação periférica. ${ }^{16}$

- Estímulos repetitivos aumentam a resposta de dor na AR

Wendler et al. (1999) estudaram as mudanças no sistema nociceptivo em indivíduos com AR em comparação com saudáveis. 0 estudo foi feito com a utilização de um eletroencefalografia e uma escala visual analógica, que mensurou a dor dos indivíduos. Os estímulos dolorosos foram dados a partir de concentrações de gás carbônico (CO2). Os dois grupos responderam da mesma maneira tanto com o aumento do estímulo doloroso a medida que foi aumentando a dose de $\mathrm{CO2}$, quanto para as respostas à diferentes ou constantes intensidades e ao obterem estímulos repetitivos com intervalo de 8 segundos. Porém, com intervalos de 2 segundos entre os estímulos, o grupo da AR obteve uma resposta muito maior, por isso os autores sugeriram mudança no processamento nociceptivo em dores crônicas inflamatórias, onde o sistema amplifica esta dor crônica. ${ }^{17}$

Em um modelo de dor geral, mas baseado em estudos com AR, Khasar et al (2005) avaliaram o efeito de sons repetitivos no limiar nociceptivo e na hiperalgesia inflamatória em animais. Foi encontrado que sons não habituais e repetitivos aumentam a hiperalgesia mecânica devido ao aumento da quantidade do mediador inflamatório bradicinina, o que não aconteceu em animais normais. Esta substância em animais normais provoca hiperalgesia pela liberação de PGE2 dos terminais nervosos simpáticos. Foi visto que o aumento da bradicinina foi dado através da contribuição da medula adrenal, pois em sua ausência houve diminuição do número de bradicinina e reversão do quadro do estresse induzido.18

- Capsaicina como marcador da alteração no processo nociceptivo

A partir de estudos que avaliaram a dor em geral e seu aumento de atividade nas vias nociceptivas, Morris em 1997 investigou se é possível quantificar as mudanças na sensibilidade neuronal em pacientes com AR com o uso da capsaicina. Em todos os indivíduos a capsaicina produziu uma sensação de pontada e calor na área da aplicação. Porém, a média de área que a hiperalgesia atingiu foi maior nos pacientes com AR. Entretanto, o tempo do curso de desenvolvimento da hiperalgesia foi similar nos dois grupos. Esses resultados evidenciam o aumento da sensibilização das fibras sensoriais em pacientes com AR. Por isso foi sugerido a presença de mecanismos centrais e não apenas periféricos nessa desordem.19

- Comparação do limiar e tolerância entre indivíduos com AR e saudáveis

Jahanshahi et al. (1989) analisaram o comportamento da dor em pacientes com AR comparando com saudáveis, através da escala visual analógica e do questionário McGill. Palavras como picada, cansativa, irritante, insuportável, ranzinza foram marcadas por $50 \%$ dos participantes, e a palavra "dor" foi a mais referida. Neste questionário os indivíduos com AR obtiveram o escore mais alto, ou seja, marcaram mais palavras. Além disso, foi observado que estes possuem menor limiar e tolerância a um teste com água gelada, quando comparados com indivíduos saudáveis. ${ }^{20}$

Partindo do princípio da teoria das comportas e que pacientes com AR possuem menor limiar de dor, Dhondt em 1999 testou uma técnica manual com o objetivo de aumentar o limiar de dor de indivíduos com AR. Na mensuração anterior à técnica, foi visto que pacientes com AR possuem um menor limiar doloroso comparado a indivíduos sadios, o que sugere alteração no sistema nociceptivo. Porém, na segunda mensuração, o limiar de dor aumentou significantemente nos pacientes com AR e imediatamente após a mobilização articular nas vértebras torácica 6 , lombares 1 e 3 em comparação com os sadios. ${ }^{21}$ 


\section{Discussão}

Esta pesquisa teve como objetivo realizar uma revisão sistemática da literatura a respeito dos principais mecanismos da dor nociceptiva da Artrite Reumatóide (AR). Foi evidenciado que fatores como TNF- $\alpha$, quimiocinas, citocinas, prostanóides, COX-2, PKC, neutrófilos e canal TRPV1 estão relacionados à manifestação álgica nociceptiva da AR.

A dor por nocicepção, principalmente a aguda, é imprescindível para a preservação da integridade do indivíduo, por ser um sintoma que alerta ocorrências de lesões no organismo. Já a dor crônica não tem este valor biológico e é uma importante causa de incapacidade.22

TNF- $\alpha$ como importante estratégia de tratamento e sua relação com prostaglandinas, canal TRPV1, PKC, COX-2 e neutrófilos

0 Fator de Necrose Tumoral-alfa (TNF- $\alpha$ ) é constantemente encontrado no líquido sinovial de articulações inflamadas em diversos cenários clínicos, por isso o estudo dessa citocina é considerado muito importante para a compreensão da fisiopatologia da AR. ${ }^{13}$ Já foi comprovada a presença de TNF- $\alpha$ nos níveis séricos de pacientes com AR, como resposta aos diversos estímulos dolorosos. ${ }^{4}$

Os resultados dessa revisão sistemática mostraram evidências de que o TNF- $\alpha$ está diretamente relacionado à dor nociceptiva da $A R$, atuando como provocador da hiperalgesia. A presença dessa citocina foi associada à ativação de mediadores como IL-1ß, prostaglandinas e PKC, que desencadeariam sensibilização de nociceptores, hiperalgesia e estimulação central. Ainda foi proposto haver uma sensibilização de canais TRPV1 via PKC, resultando em hiperalgesia, e produção de COX-2 derivada de prostaglandinas, apesar da ação desta não ser necessária para a ocorrência da hiperalgesia.13

Evidenciando a importância do TNF- $\alpha$ em processos inflamatórios em geral, a literatura demonstra que o bloqueio da ação desta citocina impede o influxo de neutrófilos e a produção de IL-1ß e que esse efeito causa diminuição da hipernocicepção. ${ }^{1}$

Esta revisão também demonstrou repercussões do TNF- $\alpha$ no sistema nervoso central, evidenciando diminuição das atividades cerebrais, após bloqueio da citocina, em áreas como: tálamo, córtex somatosensorial secundário, sistema límbico, ínsula e córtex cingulado anterior, envolvidas com percepção da dor, emoção e memória. ${ }^{13}$

Em decorrência de um estímulo de dor aguda, os pacientes com AR mostram respostas corticais e subcorticais amenizadas em comparação com pessoas sadias, como áreas do córtex pré-frontal dorsolateral e cingulado anterior ${ }^{24}$ sendo a primeira área relacionada ao comportamento executivo e auto-percepção e a segunda relacionada a emoções e comportamentos. ${ }^{25}$

Adicionalmente, pacientes com doenças inflamatórias crônicas freqüentemente apresentam sinais de fadiga e depressão 26,27 e tem sido demonstrado que citocinas circulantes e ativação de fibras sensoriais periféricas estão intimamente relacionadas a essas alterações no SNC.27 O TNF- $\alpha$ é um importante mediador dessas alterações e já foi demonstrado que a administração de anticorpos anti-TNF- $\alpha$ resultou em diminuição significativa da fadiga26, melhora na sensação de bem-estar e diminuição da dor em articulações. ${ }^{29}$ Corrobora-se, então, a idéia de que níveis elevados de TNF- $\alpha$ possuem importante impacto nas atividades cerebrais.300 TNF- $\alpha$ tem sido visto como um regulador central da inflamação e por isso técnicas que antagonizem seu efeito têm sido extensamente investigadas na terapêutica de diversas doenças inflamatórias. ${ }^{31}$ Devido aos vários aspectos envolvidos na dor, é necessário que o diagnóstico seja estabelecido o mais cedo possível, assim como as estratégias terapêuticas busquem o controle e a minimização das condições causais. ${ }^{32}$ Dessa forma, ressalta-se a importância de um tratamento multidisciplinar, em que sejam abordados os diversos cenários da dor, incluindo aspectos físicos, psíquicos e comportamentais. 


\section{Canal TRPV1}

O TRPV1 é um canal de cátion não-seletivo, expresso principalmente nos neurônios nociceptivos do gânglio da raiz dorsal. 33 Esta revisão demonstra como resultado que o aumento do TNF- $\alpha$ provoca crescimento da expressão do canal TRPV1, apontando que a ação do TNF- $\alpha$ depende deste canal.13 A literatura apresenta ainda a expressão do canal TRPV1 em fibroblastos sinoviais de pacientes com AR e comprova que o canal TRPV1 pode desempenhar um papel tanto na modulação da nocicepção na AR, como também um papel neuronal.33 Ao administrar um antagonista do canal TRPV1 foi bloqueada a ação do SA13353, um inibidor da produção de TNF- $\alpha$, ou seja, a liberação do TNF- $\alpha$ se deu a partir do antagonista do canal TRPV1, e não pelo próprio canal, discordando no que diz respeito a uma dependência do TNF$\alpha$ pelo TRPV1.34

\section{Proteína quinase C (PKC)}

Para que haja o aumento de TRPV1, é imprescindível a presença da PKC para fosforilar, potencializar13 e sensibilizar estes canais.35 Estudos descrevem a bradicinina (BK) como um peptídeo liberado por conseqüência do dano tecidual, com consequente ativação da PKC. Esta última possui cinco isoformas em neurônios sensoriais.36 Além disso, as cininas causam fosforilação da PKC que a partir daí, podem mediar a sensibilização de transdutores sensoriais como TRPV1.37

\section{Ciclo-oxigenase -2 (COX-2)}

0 atual estudo também trouxe como resultado o achado de que a COX-2 é induzida por prostaglandinas (PGE) na articulação durante o evento álgico e que esse fenômeno ocorre simultaneamente à produção de TNF- $\alpha$. Porém, foi visto que esses fatores não são essenciais para o processo de hiperalgesia.13 Contudo, estudos mostram que os prostanóides, principalmente as prostaglandinas, associadas às variações de COX, são consideradas as maiores contribuidoras para dor inflamatória. 37

A literatura nos mostra que prostaglandinas presentes da medula espinal induzem a hiperexcitabilidade com o aumento da expressão de COX-1(glia) e COX-2 (células do corno ventral da medula).37 Ao administrar um inibidor da COX, foi inibida também a interleucina 17, provocando diminuição da hiperalgesia. Associado a isso, ao administrar doses de IL-17 nos animais, pôde-se perceber um aumento da produção de COX-2 e prostaglandinas.15

Muitos estudos têm revelado que a ação de inibidores da COX-2 tem sido muito eficaz no tratamento da inflamação crônica, porém deve-se ter bastante cuidado ao ministrá-la em decorrência do seu risco de insuficiência renal e hipertensão arterial. Estes distúrbios estão diretamente relacionados às doses de inibidores da COX-2, por isso, existem boas razões para evitar altas doses, principalmente em indivíduos idosos.38

Citocinas, prostanóides, quimiocinas, neutrófilos e células da glia possuem um importante papel na hipernocicepção inflamatória

\section{Interleucina-17}

A interleucina-17 (IL-17) possui importante papel na hipernocicepção articular, havendo uma diminuição do número de neutrófilos, quando a mesma é bloqueada.15 Estudos revelam que uma adição exógena de IL-17 aumenta a produção de PGE2 e estimula as vias pró-inflamatórias, tais como a produção de citocinas inflamatórias e aumento da osteoclastogênese na AR.39 Desta forma, contribui para a cronicidade da AR, além de induzir células sinoviais a um padrão de expressão parecido ao de quando estão na presença do TNF$\alpha$. Outro autor demonstrou que as citocinas melhoraram suas respostas quando há uma estabilização da expressão de mais citocinas e quando há aumento no número dos seus receptores, além de aumentarem a expressão e a migração de genes de quimiocinas.40

\section{Prostanóides}

Nesta revisão foi observado que a administração de IL-17 promove um aumento nos níveis de PGE na articulação e que a injeção desta juntamente com dopamina provocou 
diminuição do limiar nociceptivo, ou seja, aumento da inflamação. Concluindo que os prostanóides parecem mediar a hipernocicepção na indução da artrite por IL-17.15

As prostaglandinas (PGs) são metabólitos de ácido aracdônico, encontrados em altos níveis no líquido e membrana sinovial e são conhecidas por desempenhar um papel fundamental no desenvolvimento da vasodilatação, extravazamento de líquido e dor nos tecidos sinoviais. ${ }^{41}$

A indução da hiperalgesia parece ocorrer essencialmente por duas substâncias, a prostaciclina e PGE2. A primeira induziu um efeito imediato da hiperalgesia, enquanto que a segunda obteve um longo período de latência. Observou-se também, que altas doses de PGE2 ou a administração de pequenas doses sucessivas resultaram em um efeito mais duradouro. De forma semelhante, isso pode ocorrer nas articulações com AR, devido à inflamação duradoura e com recidivas que o quadro clínico se manifesta. 42

\section{Quimiocinas}

As quimiocinas possuem grande importância na organização da migração de leucócitos para o local inflamado, mas também estão implicadas no desenvolvimento cerebral e na função sináptica. Receptores de quimiocinas estão sendo detectados em todo o SNC, nas células de macrófagos como a microglia, oligodendrócitos, neurônios aferentes primários e gânglio da raiz dorsal.37 As quimiocinas podem contribuir diretamente para a hiperalgesia através da sensibilização dos receptores dos canais como o TRPV1, além de sensibilizar também os neurônios sensoriais. ${ }^{43}$

A liberação de mediadores inflamatórios é regulada pelos receptores gliais, cininas, prostaglandinas, canal N-metil-D-aspartato (NMDA) e por vários receptores de quimiocinas que podem modular a excitabilidade neuronal. ${ }^{44}$

\section{Célula da glia e Citocinas}

A IL-17 induz a expressão de quimiocinas e também de citocinas.15 Estudos descrevem a dor através de uma reação glial caracterizada por proliferação, hipertrofia e expressão de citocinas pró-inflamatórias como IL-1ß e TNF- $\alpha$ alfa. Desta forma, pode-se considerar o envolvimento da glia no mecanismo central de manutenção e amplificação do estado da dor. Paralelamente, foi verificado no corno dorsal da medula, um aumento da coloração da microglia em momentos precoces e tardios da inflamação, enquanto que a coloração dos astrócitos aumentou apenas durante a fase inflamatória.42

As citocinas induzem a hiperalgesia por uma série de ações diretas e indiretas como, por exemplo, o IL-1ß ativa nociceptores diretamente (via intracelular de ativação de cinina) e juntamente com TNF- $\alpha$ causa sensibilização nos nociceptores (via prostanóides e cininas), via receptores TNFR1 e 2. Além disso, inicia-se uma cascata de reações inflamatórias na produção de IL-1, IL-6 E IL-8.37

- Expressão de neutrófilos durante a inflamação tecidual periférica

Esta revisão trouxe nos resultados que houve aumento da presença dos genes S100A8 e S100A9, ligados à expressão de neutrófilos e monócitos, assim como acontece na AR.17 Em estudos com atrite induzida por IL-17 também há envolvimento dos neutrófilos, sugerindo que 0 IL-17 é um potente quimio-atraente de células imunológicas como células $\mathrm{T}$ e neutrófilos17, 46, 47, além de promover a migração de bradicininas na fase crônica da inflamação sinovial46, devido a presença dos receptores de bradicinina na superfície dos neutrófilos.48

- Estímulos repetitivos aumentam a resposta de dor na AR

As pesquisas dessa revisão apontam diferentes respostas a estímulos dolorosos em indivíduos sadios e com AR. Foi visto que estímulos repetidos levam a uma exacerbação na resposta à dor nos indivíduos com AR. Sugere-se, portanto, uma alteração no processo 
nociceptivo nas dores crônicas inflamatórias desses indivíduos.18 Outros estudos mostraram que sons repetitivos e não-habituais em animais com artrite induzida19,48, provocaram um aumento da hipernocicepção causada pelo crescimento da quantidade de bradicinina49 geradas durante lesão tecidual e estímulo nocivo - 48, prostaglandinas E2, proteína-G, PKC e até mesmo a ativação das vias hipotálamo-pituitária-adrenal e simpático-adrenal. Estes fatores agem modificando a via de sinalização dos nociceptores aferentes ${ }^{19,37}$ o que não aconteceu em animais normais. ${ }^{19}$

\section{Capsaicina como marcador da alteração no processo nociceptivo}

Estudos mostram que ao injetar capsaicina nas articulações ocorrem diferentes mudanças nas respostas nociceptivas entre indivíduos sadios e com AR. Além da sensação de pontada e calor sentida pelos dois grupos, os pacientes com AR apresentaram uma maior área de hiperalgesia.20,50 Houve uma vasodilatação axonal reflexa nos dois grupos, porém o grupo com AR foi significantemente maior. Além disso, verificou-se que houve uma correlação entre a vasodilatação reflexa e a Escala Visual Analógica (EVA) apenas no grupo com AR. 50

- Comparação de limiar e tolerância entre indivíduos com AR e saudáveis

Resultados indicam que indivíduos com AR além de obter um escore maior em um questionário de avaliação da dor, obtiveram um menor limiar e tolerância em um teste com água gelada21, menor limiar na avaliação com algômetro de pressão ${ }^{51}$ e na utilização dos filamentos de Von Frey, além de maiores respostas na Escala Visual Analógica (EVA) e maiores circunferências articulares e graus de temperatura, comparado com indivíduos sadios. 52

Os pacientes acometidos por AR possuem grande limitação funcional devido à dor constante desde o início da doença, piorando progressivamente. Essa revisão mostrou que a causa da dor nociceptiva é influenciada principalmente pelos processos inflamatórios. Dessa forma, ao elucidar os mecanismos da dor nociceptiva na Artrite Reumatóide, esse trabalho indica as principais substâncias envolvidas na nocicepção, para que sejam consideradas como medidas de resultados em futuros estudos de intervenção terapêutica.

\section{Conclusão}

Este estudo teve algumas dificuldades e limitações como a falta de cegamento entre os autores na seleção dos artigos e a dificuldade de encontrar estudos que realmente abordassem o mecanismo da dor, pois a maioria elucidava os tratamentos propostos para a doença. Além disso, uma grande quantidade de estudos não descrevia a metodologia da pesquisa, ou seja, abordava aspectos teóricos sem especificar os métodos empregados sendo, portanto, excluídos da análise metodológica. Ao contrário, os artigos analisados nessa pesquisa relataram de forma clara as suas metodologias. Em decorrência de todos esses fatores, evidenciou-se uma necessidade de novos estudos que busquem se aprofundar nos mecanismos existentes na dor da Artrite Reumatóide e que apresente de forma minuciosa o método utilizado para a pesquisa.

Os mecanismos da dor nociceptiva da Artrite Reumatóide foram discutidos e pôde-se observar a relevância de algumas substâncias como o TNF- $\alpha$, a IL-17, a PKC e o canal TRPV1 para a potencialização dessa dor. Portanto, nessa revisão evidencia-se mais uma vez a necessidade de um tratamento multidisciplinar, para que possam ser abordados os diversos aspectos da dor, incluindo os seus componentes físicos, emocionais e comportamentais. Também, as intervenções multidisciplinares desde o início da doença poderá minimizar ou retardar as amplificações da dor no SNC, evitando o grande impacto na qualidade de vida e sofrimento desses pacientes. 


\section{Referências}

1. SACHS D, et al. "Cooperative role of tumour necrosis factor- $\alpha$, interleukin- $1 \beta$ and neutrophils in a novel behavioural model that concomitantly demonstrates articular inflammation and hypernociception in mice", British Journal of Pharmacology, 162(1): 72-83. 2011.

2. RADOVITS, B J; KIEVIT, W \& LAAN, R F. "Tumour necrosis factor-alpha antagonists in the management of rheumatoid arthritis in the elderly: a review of their efficacy and safety", Drugs \& Aging, 26(8): 647-64. 2009.

3. INOUE, A, et al. "The role of TNFalpha-induced adipose-related protein (TIARP) in TNFalpha dependent arthritic model--GPI-induced arthritis", Nihon Rinsho Meneki Gakkai Kaishi, 32(1): 15-19. 2009.

4. EDWARDS, R R, et al. "Enhanced reactivity to pain in patients with rheumatoid arthritis", Arthritis Research \& Therapy, 11(3): R61. 2009

5. BÉRTOlO, M. et al. "Atualização do Consenso Brasileiro no Diagnóstico e Tratamento da Artrite Reumatóide", Temas de Reumatologia Clínica, 10(1): 2009.

6. RODRIGUES, C; BÓ S; TEIXEIRA R. "Diagnóstico Precoce da Artrite Reumatóide", Revista Brasileira de Análises Clínicas, 37(4): 201-04. 2005.

7. ALETAHA, D; SMOLEN, J \& WARD M. “Measuring Function in Rheumatoid Arthritis: Identifying reversible and irreversible components", Arthritis \& Rheumatism, 54(9): 2784-92. 2006.

8. SANGHA, O. "Epidemiology of Rheumatic diseases", Rheumatology, 39(2): 3-12. 2000.

9. HAZES, J, et al. "Physical function improvements and relief from fatigue and pain are associated with increased productivity at work and at home in rheumatoid arthritis patients treated with certolizumab pegol", Rheumatology, 49(10): 1900-10. 2010.

10. McKENNA, F, \& WRIGHT, V. "Pain and rheumatoid arthritis", Annals of the Rheumatic Diseases, 44(11): 805. 1985.

11. JADAD, A, et al. "Assessing the quality of reports of randomized clinical trials: is blinding necessary?", Controlled Clinical Trials, 17(1): 1-12. 1996.

12. PAI, M, MCCULLOCH, M \& COLFORD, J. "Diagnostic systematic reviews: a road map (version 3)", Disponível em: <www.medepi.net/meta/guidelines/ Diagnostic_Systematic_Reviews_Road_Map_V3 .pdf>. Acesso em: 05/06/11).

13. RUSSEL, F, et al. “Tumour necrosis factor a mediates transient receptor potential vanilloid 1dependent bilateral thermal hyperalgesia with distinct peripheral roles of interleukin-1b, protein kinase $C$ and cyclooxygenase-2 signalling", Pain, 142(3): 264-74. 2009.

14. HESS, A, et al. "Blockade of TNF- $\alpha$ rapidly inhibits pain responses in the central nervous system", Proceedings of the National Academy of Sciences, 108(9): 3731-36. 2011.

15. PINTO, L, et al. "IL-17 mediates articular hypernociception in antigen-induced arthritis in mice", Pain, 148(2): 247-56. 2010.

16. CONSTANDIL, L, et al. "Effect of interleukin-1beta on spinal cord nociceptive transmission of normal and monoarthritic rats after disruption of glial function", Arthritis Research \& Therapy, 11(4): R105. 2009.

17. MITCHELL, K, et al. "Localization of S100A8 and S100A9 expressing neutrophils to spinal cord during peripheral tissue inflammation", Pain, 134(1-2): 216-31.2008.

18. WENDLER, J, et al. "Patients with rheumatoid arthritis adapt differently to repetitive painful stimuli compared to healthy controls", Journal of Clinical Neuroscience, 8(3): 272-77. 2001.

19. KHASAR, S, GREEN, P \& LEVINE, J. "Repeated sound stress enhances inflammatory pain in the rat", Pain, 116(1-2): 79-86. 2005.

20. MORRIS, V, CRUWYS, S \& KIDD, B. “Characterization of capsaicin-induced mechanical hyperalgesia as a marker for altered nociceptive processing in patients with rheumatoid arthritis", Pain, 71(2): 179-86. 1997. 
21. JAHANSHAHI, M, PITT P \& WILLIAMS, I. "Pain avoidance in rheumatoid arthritis", Journal of Psychosomatic Research, 33(5): 579-89. 1989.

22. DHONDT, $W$, et al. "Pain threshold in patients with rheumatoid arthritis and effect of manual oscillations", Scandinavian Journal of Rheumatology, 28(2): 88-93. 1999.

23. MUNDINGER, F \& SALOMÃO, J. "Deep brain stimulation in mesencephalic lemniscus medialis for chronic pain", Acta Neurochirurgica, 30: 245-85. 1980.

24. JONES, A \& DERBYSHIRE, S. "Reduced cortical responses to noxious heat in patients with rheumatoid arthritis", Annals of the Rheumatic Diseases, 56(10): 601-07. 1997.

25. LUNDY-EKMAN, L. Cérebro. Neurociências: fundamentos para reabilitação. Rio de Janeiro: Elsevier, 2008. p. 367.

26. WOLFE, F \& MICHAUD, K. "Fatigue, rheumatoid arthritis, and anti-tumor necrosis factor therapy: an investigation in 24,831 patients", Journal of Rheumatology, 31(11): 2115-20. 2004.

27. NAS, K, et al. "Psychological status is associated with health related quality of life in patients with rheumatoid arthritis", Journal of Back and Musculoskeletal Rehabilitation, 24(2): 95-100. 2011.

28. DANTZER, R, et al. "Cytokines and depression: fortuitous or causative association?" Molecular Psychiatry, 4(4): 328-32. 1999.

29. COHEN, O, et al. "Endotoxin-induced changes in human working and declarative memory associate with cleavage of plasma "readthrough" acetylcholinesterase", Journal of Molecular Neuroscience, 21(3): 199-212. 2003.

30. STRAUB, R, et al. “Tumor necrosis factor-neutralizing therapies improve altered hormone axes: an alternative mode of antiinflammatory action", Arthritis \& Rheumatism, 54(7): 2039-46. 2006.

31. ESPOSITO, E \& CUZZOCREA, S. "TNF-alpha as a therapeutic target in inflammatory diseases, ischemia-reperfusion injury and trauma", Current Medicinal Chemistry, 16(24): 3152-67. 2009.

32. TEIXEIRA M. "Anatomia e fisiologia das unidades nociceptivas e supressoras da dor. Dor: contexto interdisciplinar. Curitiba: Maio, 2003. p. 119.

33. ENGLER A, et al. "Expression of transient receptor potential vanilloid 1 (TRPV1) in synovial fibroblasts from patients with osteoarthritis and rheumatoid arthritis", Biochemical and Biophysical Research Communications, 359(4): 884-8. 2007.

34. MURAI, M, et al. "SA13353 (1-[2-(1-Adamantyl)ethyl]-1-pentyl-3-[3-(4-pyridyl)propyl]urea) inhibits TNF-alpha production through the activation of capsaicin-sensitive afferent neurons mediated via transient receptor potential vanilloid 1 in vivo", European Journal of Pharmacology, 588(2-3): 309-15. 2008.

35. ZHU, W, et al. "Activin acutely sensitizes dorsal root ganglion neurons and induces hyperalgesia via PKC-mediated potentiation of transient receptor potential vanilloid I", Journal of Neuroscience, 27(50): 13770-80, 2007.

36. CESARE P, et al. "Specific involvement of PKC-epsilon in sensitization of the neuronal response to painful heat", Neuron Journal, 23(3): 617-24. 1999.

37. DRAY, A, et al. "New horizons in pharmacologic treatment for rheumatic disease pain", Rheumatic Disease Clinics of North America, 34(2): 481-505. 2008.

38. SAVAGE, R, et al. "Cyclo-oxygenase-2 inhibitors: when should they be used in the elderly?" Drugs \& Aging, 22(3): 185-200. 2005.

39. ITO, H, et al. "Dual role of interleukin-17 in pannus growth and osteoclastogenesis in rheumatoid arthritis", Arthritis Research \& Therapy, 13(1): R14. 2011.

40. HOT, A \& MIOSSEC, P. "Effects of interleukin (IL)-17A and IL-17F in human rheumatoid arthritis synoviocytes", Annals of the Rheumatic Diseases, 70(5): 727-32. 2011.

41. STARÍCZYK, J \& KOWAL, M. "The role of cyclooxygenase and prostaglandins in the pathogenesis of rheumatoid arthritis", Polski merkuriusz lekarski, 11(65): 438-43. 2001. 
42. FERREIRA, S, NAKAMURA M, CASTRO, M A. "The hyperalgesic effects of prostacyclin and prostaglandin E2", Prostaglandins \& Other Lipid Mediators, 16(1): 31-37. 1978.

43. ZHANG, N, et al. "A proinflammatory chemokine, CCL3, sensitizes the heat- and capsaicin-gated ion channel TRPV1", Proceedings of the National Academy of Sciences of the United States of America, 102(12): 4536-41. 2005.

44. WATKINS, L \& MAIER, S. “Glia: a novel drug discovery target for clinical pain”, Nature Reviews Drug Discovery, 2(12): 973-85. 2003.

45. CHRISTIANSON, C, et al. "Characterization of the acute and persistent pain state present in $\mathrm{K} / \mathrm{BxN}$ serum transfer arthritis", Pain, 151(2): 394-403. 2010.

46. FERRACCIOLI, G \& ZIZZO, G. "The potential role of Th17 in mediating the transition from acute to chronic autoimmune inflammation: rheumatoid arthritis as a model", Discovery Medicine, 11(60): 413-24. 2011.

47. STRZEPA, A \& SZCZEPANIK, M. "IL-17-expressing cells as a potential therapeutic target for treatment of immunological disorders", Pharmacological Reviews, 63(1): 30-44. 2011.

48. COUTURE, R, et al. "Kinin receptors in pain and inflammation", European Journal of Pharmacology, 429(1-3): 161-76. 2001.

49. KHASAR, S, et al. "Stress induces a switch of intracellular signaling in sensory neurons in a model of generalized pain", The Journal of Neuroscience, 28(22): 5721-30. 2008.

50. JOLLIFFE, V; ANAND, P \& KIDD, B. "Assessment of cutaneous sensory and autonomic axon reflexes in rheumatoid arthritis", Annals of the Rheumatic Diseases, 54(4): 251-55. 1995.

51. FREDRIKSSON, L; ALSTERGREN, P \& KOPP S. "Pressure pain thresholds in the craniofacial region of female patients with rheumatoid arthritis", Journal of Orofacial Pain, 17(4): 326-32. 2003.

52. HENDIANI J, et al. "Mechanical sensation and pain thresholds in patients with chronic arthropathies", Pain, 4(4): 203-11. 2003. 


\section{Apêndice}

Figura 1

Critério de seleção dos artigos

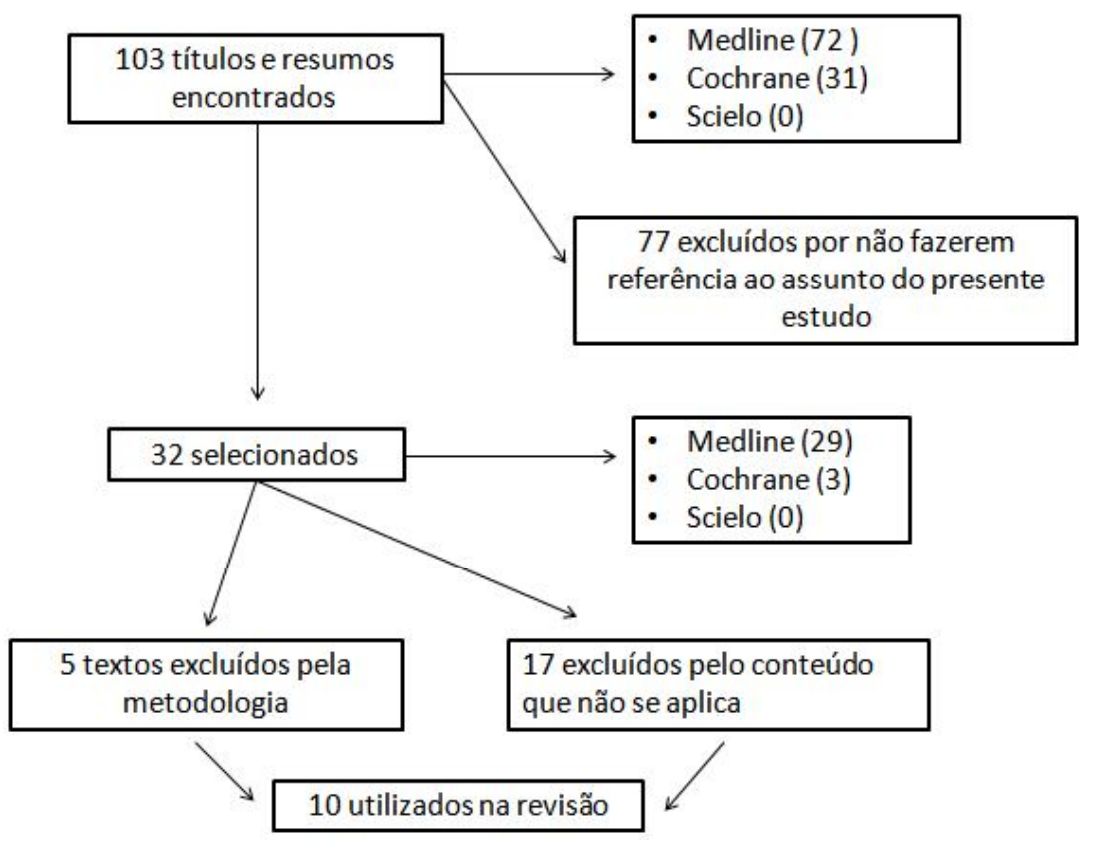

Quadro 1:

Análise metodológica dos artigos - Escala de Jadad

\begin{tabular}{|c|c|c|c|c|c|c|c|c|c|}
\hline & $\begin{array}{l}\text { Rando- } \\
\text { mização }\end{array}$ & $\begin{array}{l}\text { Cega- } \\
\text { mento }\end{array}$ & $\begin{array}{l}\text { Objeti- } \\
\text { vose } \\
\text { hipó- } \\
\text { teses } \\
\text { claras }\end{array}$ & $\begin{array}{c}\text { Compa- } \\
\text { ração } \\
\text { com } \\
\text { placebo } \\
\text { / sham }\end{array}$ & $\begin{array}{c}\text { Descri- } \\
\text { ção da } \\
\text { inter- } \\
\text { venção }\end{array}$ & $\begin{array}{c}\text { Análise } \\
\text { ade- } \\
\text { quada }\end{array}$ & $\begin{array}{l}\text { Descri- } \\
\text { ção do } \\
\text { método }\end{array}$ & $\begin{array}{l}\text { Obje- } \\
\text { tivos } \\
\text { Alcan- } \\
\text { çados }\end{array}$ & Escore \\
\hline $\begin{array}{c}\text { Hess, A.; et al, } \\
2011\end{array}$ & & $\mathrm{X}$ & $\mathrm{X}$ & & $\mathrm{X}$ & $\mathrm{X}$ & $\mathrm{X}$ & $\mathrm{X}$ & ++++++ \\
\hline $\begin{array}{c}\text { Pinto, L; et al, } \\
2010\end{array}$ & & & $\mathrm{X}$ & & $\mathrm{X}$ & $\mathrm{X}$ & $\mathrm{X}$ & $\mathrm{X}$ & +++++ \\
\hline $\begin{array}{c}\text { Constandil, L. et } \\
\text { al, } 2009\end{array}$ & & & $\mathrm{X}$ & $\mathrm{X}$ & $\mathrm{X}$ & $\mathrm{X}$ & $\mathrm{X}$ & $\mathrm{x}$ & ++++++ \\
\hline $\begin{array}{c}\text { Russel, F.; et al, } \\
2009\end{array}$ & & $\mathrm{X}$ & $\mathrm{X}$ & & $\mathrm{X}$ & $\mathrm{X}$ & $\mathrm{X}$ & $\mathrm{X}$ & ++++++ \\
\hline $\begin{array}{l}\text { Mitchell, K.; et } \\
\text { al, } 2008\end{array}$ & & & $\mathrm{X}$ & & $\mathrm{X}$ & $\mathrm{X}$ & $\mathrm{X}$ & $\mathrm{X}$ & +++++ \\
\hline $\begin{array}{c}\text { Khasar, S.; et al, } \\
2005\end{array}$ & & & $\mathrm{X}$ & $\mathrm{X}$ & $\mathrm{X}$ & $\mathrm{X}$ & $\mathrm{X}$ & $\mathrm{X}$ & ++++++ \\
\hline $\begin{array}{l}\text { Wendler, J.; et } \\
\quad \text { al, } 1999\end{array}$ & $\mathrm{X}$ & & $\mathrm{X}$ & $\mathrm{X}$ & $\mathrm{X}$ & $\mathrm{X}$ & $\mathrm{X}$ & $\mathrm{X}$ & +++++++ \\
\hline
\end{tabular}




\begin{tabular}{|c|c|c|c|c|c|c|c|c|c|}
\hline $\begin{array}{c}\text { Dhondt, W.; et } \\
\text { al, 1999 }\end{array}$ & $\mathrm{X}$ & $\mathrm{X}$ & $\mathrm{X}$ & $\mathrm{X}$ & $\mathrm{X}$ & $\mathrm{X}$ & $\mathrm{X}$ & $\mathrm{X}$ & ++++++++ \\
\hline $\begin{array}{c}\text { Morris, V; } \\
\begin{array}{c}\text { Cruwys, S.; Kidd, } \\
\text { B. 1997 }\end{array}\end{array}$ & $\mathrm{X}$ & $\mathrm{X}$ & $\mathrm{X}$ & $\mathrm{X}$ & $\mathrm{X}$ & $\mathrm{X}$ & $\mathrm{X}$ & $\mathrm{X}$ & ++++++++ \\
\hline $\begin{array}{c}\text { Jahanshahi, M; } \\
\text { Pitt, P.; } \\
\text { Williams, I. 1989 }\end{array}$ & $\mathrm{X}$ & $\mathrm{X}$ & $\mathrm{X}$ & $\mathrm{X}$ & $\mathrm{X}$ & $\mathrm{X}$ & $\mathrm{X}$ & +++++++ \\
\hline
\end{tabular}

OBS:(+) Escore dado pelo autor, em escala de avaliação de uma a oito cruzes de acordo com a presença dos itens citados, referente à qualidade do artigo.

Tabela 1:

Caracterização dos estudos - fisiopatologia AR

\begin{tabular}{|c|c|c|c|c|c|c|}
\hline AUTOR & $\begin{array}{l}\text { REVIST } \\
\text { A } \\
\text { PRODU } \\
\text { ZIDA }\end{array}$ & ANO & $\begin{array}{l}\text { TIPO } \\
\text { DE } \\
\text { ESTUD } \\
0\end{array}$ & OBJETIVO & RESULTADO & CONCLUSÃO \\
\hline $\begin{array}{l}\text { Hess, } \\
\text { A.; et al }\end{array}$ & PNAS & 2011 & $\begin{array}{l}\text { Experi- } \\
\text { mental }\end{array}$ & $\begin{array}{l}\text { Estudar os efeitos dos } \\
\text { bloqueador de TNF-alfa } \\
\text { na dor através do SNC }\end{array}$ & $\begin{array}{l}\text { Na fMRI, pôde-se perceber a } \\
\text { diminuição do fluxo } \\
\text { sanguíneo para as áreas } \\
\text { envolvidas na percepção da } \\
\text { dor como, sistema límbico, } \\
\text { córtex cingulado e insular }\end{array}$ & $\begin{array}{l}\text { Os resultados sugerem } \\
\text { que a neutralização de } \\
\text { TNF-alfa afeta a } \\
\text { atividade cerebral } \\
\text { nociceptiva no contexto } \\
\text { de artrite, como um } \\
\text { efeito antiinflamatório }\end{array}$ \\
\hline $\begin{array}{l}\text { Pinto, } \\
\text { L; et al }\end{array}$ & Pain & 2010 & $\begin{array}{l}\text { Experi- } \\
\text { mental }\end{array}$ & $\begin{array}{l}\text { Observar o papel da IL- } \\
17 \text { na nocicepção } \\
\text { articular em um modelo } \\
\text { de } \Lambda R \text { induzida }\end{array}$ & $\begin{array}{l}\text { Foi observado a } \\
\text { participação do IL-17 na } \\
\text { gênese da nocicepção } \\
\text { articular em um modelo de } \\
\text { AR mBSA induzida. A } \\
\text { indução do quadro de AR } \\
\text { foi associada ao aumento da } \\
\text { expressão de IL-17 e a } \\
\text { administração de anti-IL-17 } \\
\text { diminuiu a hipernocicepção } \\
\text { e o recrutamento } \\
\text { neutrofílico dessa condição. } \\
\text { A injeção intra-articular de } \\
\text { IL-17 também induziu } \\
\text { hipernocicepção, o } \\
\text { recrutamento neutrofílico, } \\
\text { além de induzir a expressão } \\
\text { e ser bloqueada pela } \\
\text { inibição de TNF- } \alpha \text {, IL-1ß e } \\
\text { CXCR1/2 }\end{array}$ & $\begin{array}{l}\text { A IL-17 parece ser um } \\
\text { importante alvo } \\
\text { terapêutico no manejo } \\
\text { da dor e da inflamação } \\
\text { na } \Lambda \mathrm{R}\end{array}$ \\
\hline $\begin{array}{l}\text { Consta } \\
\text { ndil, L. } \\
\text { et al }\end{array}$ & $\begin{array}{l}\text { Arthriti } \\
\text { S } \\
\text { Resear } \\
\text { ch\&Th } \\
\text { erapy }\end{array}$ & 2009 & $\begin{array}{l}\text { Experi- } \\
\text { mental }\end{array}$ & $\begin{array}{l}\text { Investigar a } \\
\text { contribuição das células } \\
\text { da glia e os efeitos do } \\
\text { IL-1ß na transmissão da } \\
\text { informação nociceptiva } \\
\text { na medula espinal, em } \\
\text { um modelo de AR e um } \\
\text { placebo }\end{array}$ & $\begin{array}{l}\text { A inibição glial converteu o } \\
\text { efeito excitatório da } \\
\text { administração intra-tecal } \\
\text { de IL-1ß em um efeito } \\
\text { inibitório da nocicepção. } \\
\text { Esses efeitos foram iguais } \\
\text { para as condições de AR } \\
\text { induzida e controle. }\end{array}$ & $\begin{array}{l}\text { O efeito excitatório da } \\
\text { IL-1ß nas vias } \\
\text { nociceptivas } \\
\text { intramedulares depende } \\
\text { de mediadores gliais. O } \\
\text { estudo não conseguiu } \\
\text { demonstrar diferenças } \\
\text { entre o papel da glia ou } \\
\text { interação da IL-1B com } \\
\text { a transmissão } \\
\text { nociceptiva na } A R, \\
\text { comparado com }\end{array}$ \\
\hline
\end{tabular}




\begin{tabular}{|c|c|c|c|c|c|c|}
\hline & & & & & & indivíduos sem a doença \\
\hline $\begin{array}{l}\text { Russel, } \\
\text { F.; et al }\end{array}$ & Pain & 2009 & $\begin{array}{l}\text { Experi- } \\
\text { mental }\end{array}$ & $\begin{array}{l}\text { Elucidar os mecanismos } \\
\text { periféricos envolvidos } \\
\text { na hiperalgesia induzida } \\
\text { pelo TNF-alfa }\end{array}$ & $\begin{array}{l}\text { TNF- } \alpha \text { administrada } \\
\text { unilateralmente na região } \\
\text { plantar de camundongos } \\
\text { induziu hiperalgesia } \\
\text { bilateral, um efeito que foi } \\
\text { dependente: da PGE via PKC } \\
\text { que é dependente do } \\
\text { TRPV1, da COX2 (derivando } \\
\text { PGE) e da hipótese de que a } \\
\text { IL-1ß desempenha seu } \\
\text { papel no início da indução } \\
\text { da TNF-alfa. }\end{array}$ & $\begin{array}{l}\text { Foi comprovado que a } \\
\text { TNF-alfa envolve uma } \\
\text { cascata de eventos que } \\
\text { leva a hiperalgesia } \\
\text { bilateral, achado } \\
\text { importante para a } \\
\text { compreensão do } \\
\text { mecanismo de doença } \\
\text { em que a dor } \\
\text { inflamatória } \\
\text { desempenha um papel } \\
\text { importante, } \\
\text { especialmente no } \\
\text { contexto da } A R .\end{array}$ \\
\hline $\begin{array}{l}\text { Mitchel } \\
\text { l, K.; et } \\
\text { al }\end{array}$ & Pain & 2008 & $\begin{array}{l}\text { Experi- } \\
\text { mental }\end{array}$ & $\begin{array}{l}\text { Localizar e estudar } \\
\text { possíveis funções de } \\
\text { genes cuja a expressão é } \\
\text { regulada por um estado } \\
\text { inflamatório periférico }\end{array}$ & $\begin{array}{l}\text { Foi detectado aumento da } \\
\text { expressão dos genes } \\
\text { S100A8 e } \$ 100 \AA 9 \text { em } \\
\text { neutrófilos presentes nos } \\
\text { vasos de estruturas como } \\
\text { medula espinal, cérebro e } \\
\text { glândula pituitária, durante } \\
\text { inflamação tecidual } \\
\text { periférica }\end{array}$ & $\begin{array}{l}\text { O presente trabalho não } \\
\text { conseguiu estabelecer o } \\
\text { papel da população de } \\
\text { neutrófilos identificada } \\
\text { na nocicepção, mas } \\
\text { demonstrou que } \\
\text { neutrófilos, com } \\
\text { aumento da expressão } \\
\text { de genes implicados na } \\
\text { patologia da AR estão } \\
\text { presentes no sistema } \\
\text { vascular do SNC, o que } \\
\text { torna possível sua } \\
\text { participação na } \\
\text { inflamação }\end{array}$ \\
\hline $\begin{array}{l}\text { Khasar, } \\
\text { S.; et al }\end{array}$ & Pain & 2005 & $\begin{array}{l}\text { Experi- } \\
\text { mental }\end{array}$ & $\begin{array}{l}\text { Testar a hipótese de que } \\
\text { repetitivos sons podem } \\
\text { aumentar a mecânica da } \\
\text { dor }\end{array}$ & $\begin{array}{l}\text { Os resultados sugerem que } \\
\text { a indução do estresse } \\
\text { aumenta a hiperalgesia } \\
\text { inflamatória induzida por } \\
\text { um potente mediador } \\
\text { inflamatório- BRADICININA }\end{array}$ & $\begin{array}{l}\text { Foi demonstrado que } \\
\text { som não habitual } \\
\text { repetitivo aumenta o } \\
\text { estresse e a hiperalgesia } \\
\text { através do aumento do } \\
\text { mecanismo da } \\
\text { bradicinina }\end{array}$ \\
\hline $\begin{array}{l}\text { Wendle } \\
\text { r, J.; et } \\
\text { al }\end{array}$ & $\begin{array}{l}\text { Journal } \\
\text { of } \\
\text { Clinical } \\
\text { Neuro- } \\
\text { science }\end{array}$ & 1999 & $\begin{array}{l}\text { Ensaio- } \\
\text { Clínico }\end{array}$ & $\begin{array}{l}\text { Avaliar se há diferenças } \\
\text { entre o padrão de } \\
\text { resposta } \\
\text { eletroencefalográfica à } \\
\text { situação de dor induzida } \\
\text { entre pacientes com AR } \\
\text { e pessoas saudáveis }\end{array}$ & $\begin{array}{l}\text { Nos grupos a resposta a } \\
\text { diferentes intensidades de } \\
\text { dor e constantes } \\
\text { intensidades de dor foi a } \\
\text { mesma. Porém, quando } \\
\text { foram administrados } \\
\text { estímulos repetitivos a } \\
\text { resposta à dor foi } \\
\text { significantemente maior em } \\
\Lambda \mathrm{R}\end{array}$ & $\begin{array}{l}\text { A } \Lambda \mathrm{R} \text { altera o } \\
\text { processamento da dor, } \\
\text { de modo a amplificar a } \\
\text { resposta a um estímulo } \\
\text { nociceptivo. }\end{array}$ \\
\hline $\begin{array}{l}\text { Dhondt } \\
\text {, W.; et } \\
\text { al }\end{array}$ & $\begin{array}{l}\text { Scand J } \\
\text { Rheu- } \\
\text { matol }\end{array}$ & 1999 & $\begin{array}{l}\text { Ensaio- } \\
\text { Clínico }\end{array}$ & $\begin{array}{l}\text { Testar a hipótese de que } \\
\text { oscilação manual - uma } \\
\text { técnica de mobilização } \\
\text { passiva e rítmica- pode } \\
\text { inibir a ação nociceptiva } \\
\text { e/ou atividade } \\
\text { simpática. }\end{array}$ & $\begin{array}{l}\text { A primeira mensuração do } \\
\text { limiar mostrou que } \\
\text { pacientes com AR possuem } \\
\text { um menor limiar } \\
\text { comparado com os } \\
\text { indivíduos sadios. O que } \\
\text { sugere uma alteração no } \\
\text { sistema de processamento } \\
\text { nociceptivo. } \\
\text { Na segunda mensuração, o } \\
\text { limiar de dor aumentou } \\
\text { significativamente nos }\end{array}$ & $\begin{array}{l}\text { Novos estudos têm que } \\
\text { ser feitos, para observar } \\
\text { o efeito do limiar mais } \\
\text { tardiamente à } \\
\text { mobilização. }\end{array}$ \\
\hline
\end{tabular}




\begin{tabular}{|c|c|c|c|c|c|c|}
\hline & & & & & $\begin{array}{l}\text { pacientes com AR, depois } \\
\text { da mobilização nas áreas } \\
\text { T6, L1 e L3 em comparação } \\
\text { com os indivíduos com AR } \\
\text { que descansaram. }\end{array}$ & \\
\hline $\begin{array}{l}\text { Morris, } \\
\text { V; } \\
\text { Cruwys } \\
\text {, S.; } \\
\text { Kidd, } \\
\text { B. }\end{array}$ & Pain & 1997 & $\begin{array}{l}\text { Ensaio- } \\
\text { Clínico }\end{array}$ & $\begin{array}{l}\text { Saber se com o uso da } \\
\text { capsaicina consegue-se } \\
\text { quantificar mudanças } \\
\text { sensoriais dos } \\
\text { neurônios em pacientes } \\
\text { com } A R\end{array}$ & $\begin{array}{l}\text { O desenvolvimento da } \\
\text { hiperalgesia obteve um } \\
\text { curso parecido entre } \\
\text { indivíduos com } \Lambda \text { R e aquele } \\
\text { sadios. Os resultados } \\
\text { evidenciam o aumento da } \\
\text { sensibilização das fibras } \\
\text { sensoriais na AR, sugerindo } \\
\text { a presença de mecanismos } \\
\text { centrais nesta doença e não } \\
\text { apenas periféricos }\end{array}$ & $\begin{array}{l}\text { Os achados sugerem que } \\
\text { a capsaicina pode ser } \\
\text { usada para quantificar } \\
\text { as mudanças de } \\
\text { sensibilidade neuronal } \\
\text { em pacientes com } \mathrm{AR}\end{array}$ \\
\hline $\begin{array}{l}\text { Jahans- } \\
\text { hahi, } \\
\text { M; Pitt, } \\
\text { P.; } \\
\text { Willia } \\
\text { ms, I. }\end{array}$ & $\begin{array}{l}\text { Journal } \\
\text { of } \\
\text { Psycho } \\
\text { somati } \\
\text { c } \\
\text { Resear } \\
\text { ch }\end{array}$ & 1989 & $\begin{array}{l}\text { Ensaio- } \\
\text { Clínico }\end{array}$ & $\begin{array}{l}\text { Analisar a dor na } A R \\
\text { comparado a saudáveis } \\
\text { quanto aos termos } \\
\text { tolerância/limiar a uma } \\
\text { intensidade da dor ao } \\
\text { frio }\end{array}$ & $\begin{array}{l}\text { Dos pacientes avaliados, } \\
\text { pôde-se observar que um } \\
\text { novo estímulo nociceptivo } \\
\text { provoca significativamente } \\
\text { maior dor. O estimulo } \\
\text { doloroso foi mais relatado } \\
\text { nos componentes afetivo e } \\
\text { sensorial. Dor foi a palavra } \\
\text { mais frequente. Pacientes } \\
\text { com AR obtiveram maior } \\
\text { escore no questionário } \\
\text { McGill e menor limiar e } \\
\text { tolerância }\end{array}$ & $\begin{array}{l}\text { A cronicidade da dor, } \\
\text { talvez seja o fator mais } \\
\text { importante em } \\
\text { determinar a } \\
\text { experiência e o } \\
\text { comportamento da dor, } \\
\text { do que a dor especifica }\end{array}$ \\
\hline
\end{tabular}

FONTE: Compilação das autoras 